University of New Orleans

ScholarWorks@UNO

7-1-1991

\title{
Transmission ellipsometry on transparent unbacked or embedded thin films with application to soap films in air
}

R. M.A. Azzam

University of New Orleans, razzam@uno.edu

Follow this and additional works at: https://scholarworks.uno.edu/ee_facpubs

Part of the Electrical and Electronics Commons

\section{Recommended Citation}

R. M. A. Azzam, "Transmission ellipsometry on transparent unbacked or embedded thin films with application to soap films in air," Appl. Opt. 30, 2801-2806 (1991)

This Article is brought to you for free and open access by the Department of Electrical Engineering at ScholarWorks@UNO. It has been accepted for inclusion in Electrical Engineering Faculty Publications by an authorized administrator of ScholarWorks@UNO. For more information, please contact scholarworks@uno.edu. 


\title{
Transmission ellipsometry on transparent unbacked or embedded thin films with application to soap films in air
}

\author{
R. M. A. Azzam
}

\begin{abstract}
The ratio $\rho_{t}=T_{p} / T_{s}$ of the complex amplitude transmission coefficients for the $p$ and $s$ polarizations of a transparent unbacked or embedded thin film is examined as a function of the film thickness-to-wavelength ratio $d / \lambda$ and the angle of incidence $\phi$ for a given film refractive index $N$. The maximum value of the differential transmission phase shift (or retardance), $\Delta_{t}=\arg \rho_{t}$, is determined, for given $N$ and $\phi$, by a simple geometrical construction that involves the iso- $\phi$ circle locus of $\rho_{t}$ in the complex plane. The upper bound on this maximum equals $\arctan \{[N-(1 / N)] / 2\}$ and is attained in the limit of grazing incidence. An analytical noniterative method is developed for determining $N$ and $d$ of the film from $\rho_{t}$ measured by transmission ellipsometry (TELL) at $\phi=45^{\circ}$. An explicit expression for $\Delta_{t}$ of an ultrathin film, $d / \lambda \ll 1$, is derived in product form that shows the dependence of $\Delta_{t}$ on $N, \phi$, and $d / \lambda$ separately. The angular dependence is given by an obliquity factor, $f_{o}(\phi)=2^{1 / 2} \sin \phi \tan \phi$, which is verified experimentally by TELL measurements on a stable planar soap film in air at $\lambda=633 \mathrm{~nm}$. The singularity of $f_{o}$ at $\phi=90^{\circ}$ is resolved; $\Delta_{t}$ is shown to have a maximum just short of grazing incidence and drops to 0 at $\phi=90^{\circ}$. Because $N$ and $d / \lambda$ are inseparable for an ultrathin film, $N$ is determined by a Brewster angle measurement and $d / \lambda$ is subsequently obtained from $\Delta_{t}$. Finally, the ellipsometric function in reflection $\rho_{r}$ is related to that in transmission $\rho_{t}$.
\end{abstract}

\section{Introduction}

I have shown previously ${ }^{1}$ that the thickness $d$ and optical properties (real and imaginary parts of the complex refractive index $n-j k$ or complex dielectric function $\epsilon_{r}-j \epsilon_{i}$ ) of an unbacked or embedded thin film can be determined analytically by combined reflection and transmission ellipsometry at a suitable angle of incidence (AOI $\geq 45^{\circ}$ ). This is the only case that I know of for which the inverse problem ${ }^{2}$ of obtaining the three parameters $(n, k, d)$ of an absorbing thin film from ellipsometric data is solved without numerical iteration.

In this paper I consider the special case of a transparent $\left(k, \epsilon_{i}=0\right)$ unbacked or embedded thin film. Because only two film parameters $(n, d)$ need be determined, transmission ellipsometry (TELL) alone should suffice. And because the film is surrounded by

The author is with University of New Orleans, Lakefront, Department of Electrical Engineering, New Orleans, Louisiana 70148.

Received 13 July 1990.

0003-6935/91/192801-06\$05.00/0.

(C) 1991 Optical Society of America. transparent media of the same refractive index, straight through TELL, without beam deviation, simplifies the ellipsometer setup.

In Sec. II the behavior of the ellipsometric function $\rho_{t}=T_{p} / T_{s}$, which is the ratio of the complex amplitude transmission coefficients for the $p$ and $s$ linear polarizations parallel and perpendicular to the plane of incidence, is studied by constructing its constant angle-of-incidence contours (CAICs) and constant thickness contours (CTCs) in the complex plane for a given film refractive index. The CAICs provide a simple geometrical interpretation of the maximum differential transmission phase shift, $\Delta_{t}=\arg \rho_{t}$, that can be attained at a given AOI and the upper bound on that maximum which occurs in the limit of grazing incidence.

In Sec. III an explicit analytical method for determining $n$ and $d$ of the film from TELL at $45^{\circ}$ AOI is presented.

In Sec. IV a simple expression is derived for $\Delta_{t}$ of an ultrathin film $(d / \lambda \ll 1$, where $\lambda$ is the wavelength of light) which is tested experimentally by performing TELL on a stable soap film in air at different angles of incidence and $\lambda=633 \mathrm{~nm}$.

Section V is a brief summary of this paper.

In an appendix the ellipsometric parameters in re- 
flection are related to those in transmission for the same film at the same AOI.

\section{Ellipsometric Function}

Consider the transmission at an oblique AOI $\phi$ of a collimated beam of monochromatic or quasimonochromatic light by a plane parallel thin film of thickness $d$ (medium 1) which is sandwiched between two transparent media (denoted by 0 ) of the same refractive index, Fig. 1. The film may be unbacked, such as a soap film or a thin gold foil in air, or may be deposited on a transparent substrate and immersed in an index matching liquid. All media are assumed to be linear, homogeneous, isotropic, and nonmagnetic. The change in the state of polarization of light upon transmission through the film is determined by the ellipsometric function $\rho_{t}$ which is given by ${ }^{1}$

$$
\rho_{t}=\left[\left(1-r_{p}^{2}\right) /\left(1-r_{s}^{2}\right)\right]\left[\left(1-r_{s}^{2} X\right) /\left(1-r_{p}^{2} X\right)\right],
$$

where

$$
\begin{aligned}
& X=\exp \left[-j 4 \pi(d / \lambda) S_{1}\right], \\
& S_{1}=\left(N_{1}^{2}-N_{0}^{2} \sin ^{2} \phi\right)^{1 / 2} .
\end{aligned}
$$

$N_{1}$ and $N_{0}$ are the refractive indices of the film and surrounding medium, respectively, and $r_{p}$ and $r_{s}$ are the complex amplitude Fresnel reflection coefficients at the ambient-film (0-1) interface for the $p$ and $s$ polarizations, respectively. ${ }^{2}$

We will assume that

$$
N_{1}>N_{0} \sin \phi \text {, }
$$

so that total internal reflection does not take place at the ambient-film interface, hence $S_{1}, r_{p}$ and $r_{s}$ are real and $X$ becomes a pure phase factor:

$$
X=\exp (-j 2 \pi \zeta) \text {. }
$$

$\zeta$ is the normalized film thickness,

$$
\zeta=d / D_{\phi} \text {, }
$$

where

$$
D_{\phi}=\lambda / 2 S_{1}
$$

is the AOI-dependent film thickness period.

For a given value of the relative refractive index

$$
N=N_{1} / N_{0} \text {, }
$$

and a given AOI $\phi$, the locus of $X$, as $\zeta$ is increased from 0 to 1 (or $d$ is increased from 0 to $D_{\phi}$ ), is the unit circle in the complex plane. Because $\rho_{t}$ is related to $X$ by a bilinear transformation ${ }^{3}$ (BT), Eq. (1), its locus in the complex plane, the so-called polar curve or CAIC, is also a circle. ${ }^{4}$ Figure 2 shows a family of CAICs for a glass layer in air $(N=1.5)$ that correspond to discrete values of $\phi$ from 10 to $80^{\circ}$ in steps of $10^{\circ}$. These circles represent multiple images of the unit circle of $X$ through the BT of Eq. (1) whose coefficients vary with $\phi$.

Each circle CAIC is centered on the real axis since the substitution of $X$ by $X^{*}$ (obtained by replacing $\zeta$ by $1-\zeta)$ changes $\rho_{t}$ to $\rho_{t}^{*}$ [the coefficients of the BT are

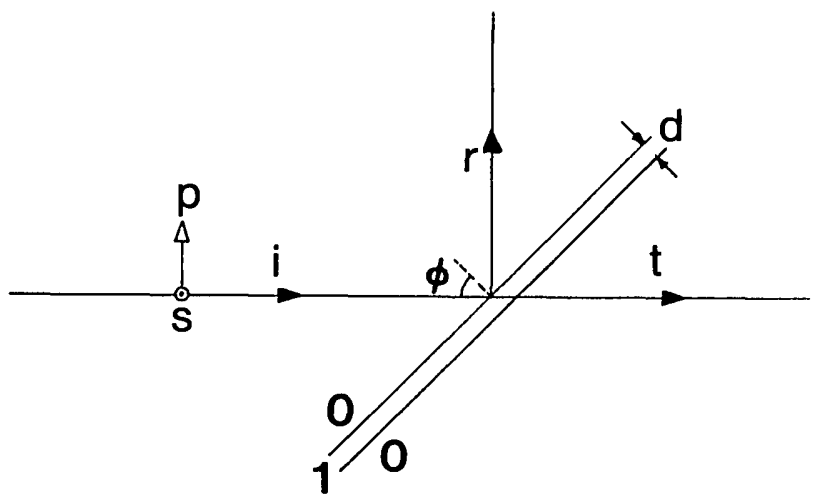

Fig. 1. Reflection and transmission of light by an unbacked or embedded thin film at oblique incidence.

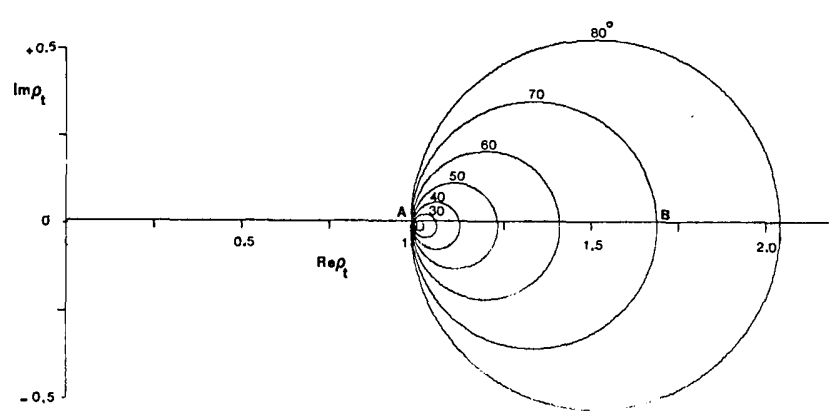

Fig. 2. Constant-angle-of-incidence contours (CAICs) of the transmission ellipsometric function $\rho_{t}$ for a glass film $(N=1.5)$ in air as a coaxial family of circles in the complex plane. The angle of incidence $\phi$ takes values from 10 to $80^{\circ}$ in steps of $10^{\circ}$.

real under the condition given by Eq. (4)]. Thus each circle CAIC is specified completely by its two points of intersection with the real axis,

$$
\begin{aligned}
& \rho_{t}^{+}=1, \\
& \rho_{t}^{-}=\left[\left(1-r_{p}^{2}\right) /\left(1+r_{p}^{2}\right)\right] /\left[\left(1-r_{s}^{2}\right) /\left(1+r_{s}^{2}\right)\right] .
\end{aligned}
$$

These points are denoted by $A$ and $B$ in Fig. 2 and represent the images of the points $X=+1$ and $X=-1$, respectively. Equation (9) corresponds to an invisible film of integral multiple of the halfwave thickness $(\zeta=$ $m$, an integer) and Eq. (10) applies to a film whose thickness is an odd multiple of a quarterwave $(\zeta=m+$ $1 / 2)$. Point $A$ is common to all CAICs which, therefore, become a coaxial family of circles. ${ }^{5}$ The term $\rho_{t}^{-}$, and the circle radius, increase monotonically with $\phi$. At normal incidence, $\phi=0, \overrightarrow{\rho_{t}}=1$ and the circle radius is 0 ; i.e., $\rho_{t}$ dwells at 1 independent of film thickness, as may be expected. We find that the grazing incidence limit of $\rho_{t}^{-}=N^{2}=2.25$.

At a given $\phi$, the maximum value, $\Delta_{t \max }$, of the differential transmission phase shift, $\Delta_{t}=\arg \rho_{t}$, is represented by the angle between the tangent line OT drawn from the origin $O$ to the CAIC circle and the real axis. From the simple geometry of Fig. 3, we have

$$
\tan \Delta_{t \max }=|C T| /|O T|=|C T| /(|O A||O B|)^{1 / 2}=|C T| /|O B|^{1 / 2},
$$

where $C$ is the circle center and $|P Q|$ represents the length of the straightline segment joining any two 


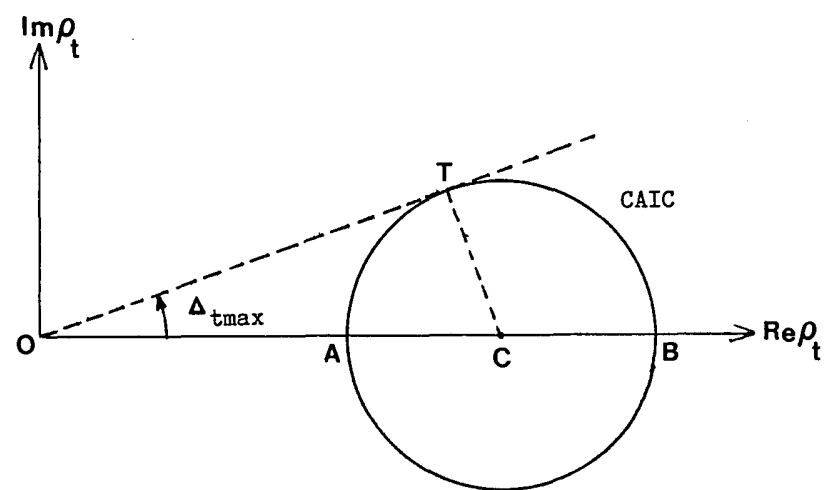

Fig. 3. Geometrical construction for finding the maximum differential transmission phase retardance, $\Delta_{t \max }$, associated with the passage of light through a transparent film (or dielectric slab) of a given refractive index and at a given angle of incidence. See text.

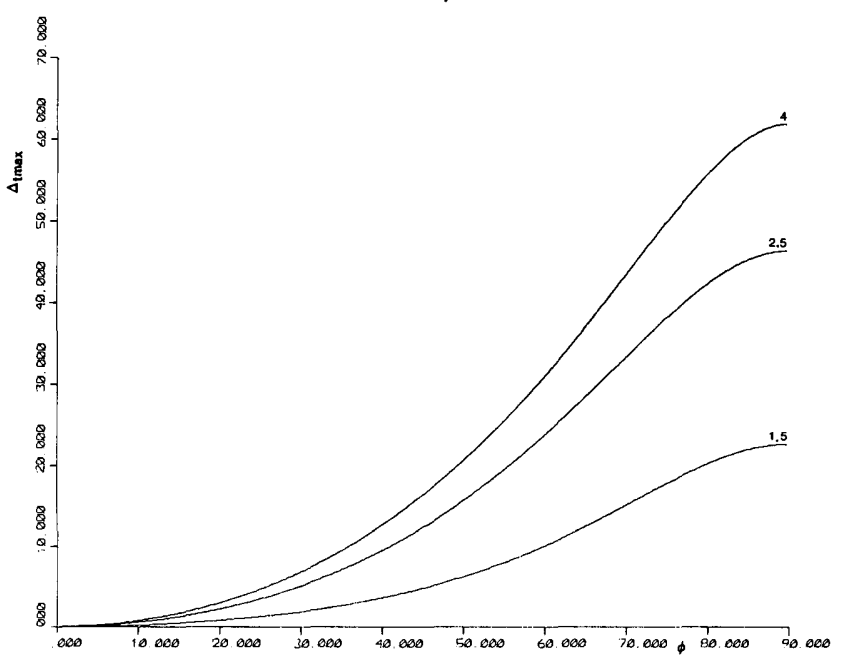

Fig. 4. Maximum transmission phase retardance, $\hat{\Delta}_{t \max }$, of a transparent film or dielectric slab as a function of the angle of incidence $\phi$ for three values of the film refractive index: $1.5,2.5$, and 4 .

points $P$ and $Q$. From Eqs. (10) and (11), we readily obtain

$$
\Delta_{t \max }=\arctan \left[\left(r_{s}^{2}-r_{p}^{2}\right) /\left(1-r_{s}^{4}\right)^{1 / 2}\left(1-r_{p}^{4}\right)^{1 / 2}\right] .
$$

Figure 4 shows $\Delta_{t \max }$ vs $\phi$ calculated from Eq. (12) for $N$ $=1.5$ (glass film in air), and for two other values of $N=$ 2.5 and 4 (Ge film in air in the IR). These results agree with those obtained by Holmes ${ }^{6}$ (based on a different formulation) who suggested the use of a tilted dielectric slab as a compensator for the analysis of polarized light.

An advantage of the analysis of this section and the construction of Fig. 3 is that it also leads to a simple expression for the upper bound, $\hat{\Delta}_{t \max }$, for the phase retardance at a given $N$, which is attainable in the limit of grazing incidence. In this case, $|C T|=\left(N^{2}-1\right) / 2$, and $|O T|=N$, so that

$$
\hat{\Delta}_{t \max }=\arctan \{[N-(1 / N)] / 2\} \text {. }
$$

Figure 5 shows a graph of this function. It is evident from this graph and from Eq. (13) that a $90^{\circ}$ retar-

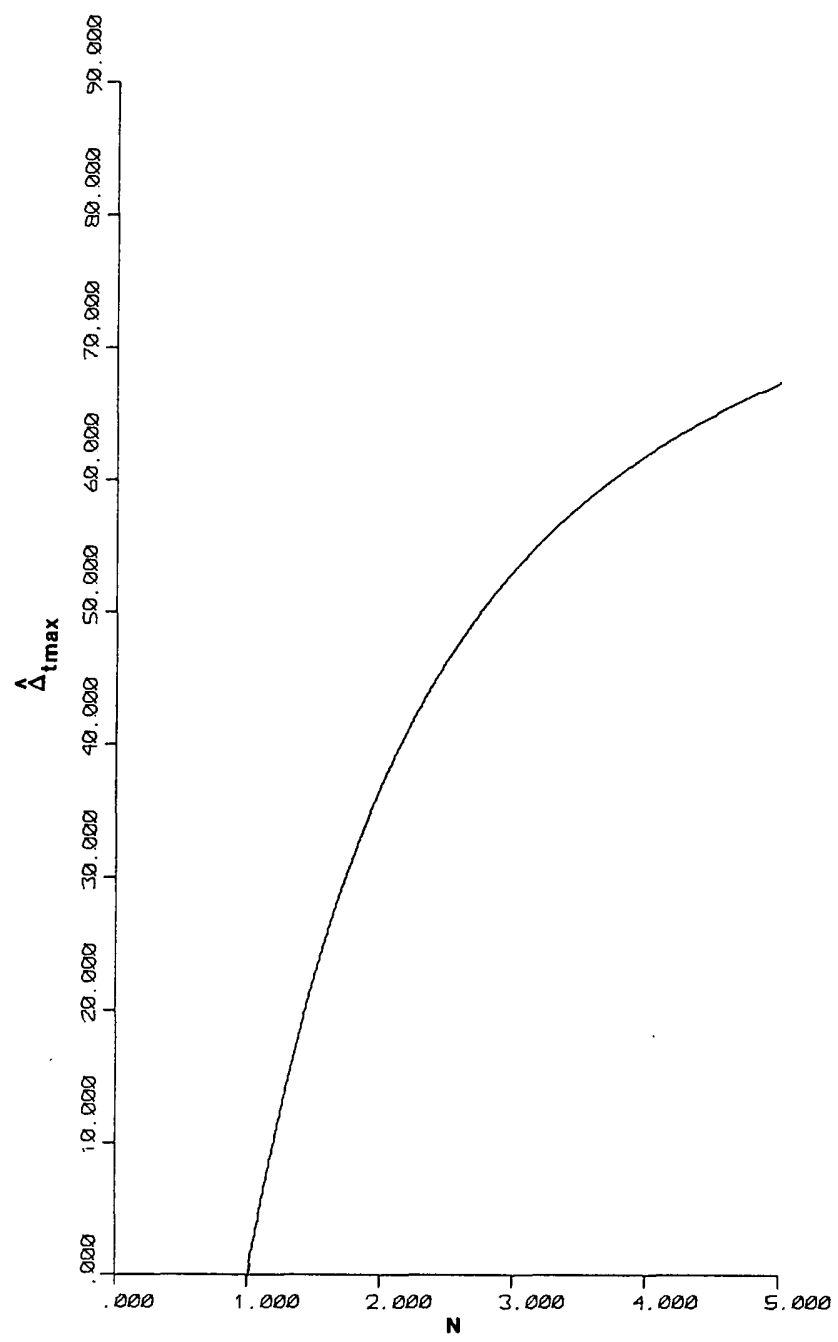

Fig. 5. Upper bound on the transmission phase retardance, $\hat{\Delta}_{t \max }$, in the limit of grazing incidence, for a transparent film or dielectric slab as a function of the film refractive index $N$.

dance is impossible to realize, hence a single slab cannot perform as a quarterwave compensator. ${ }^{6}$

The least normalized film thickness $\zeta$ required to achieve $\Delta_{t \max }$ for a specified $N$ and $\phi$ is given by

$$
\zeta=(1 / 2 \pi) \arccos \left[\left(r_{p}^{2}+r_{s}^{2}\right) /\left(1+r_{p}^{2} r_{s}^{2}\right)\right]
$$

and is plotted vs $\phi$ in Fig. 6 for $N=1.5,2.5$, and 4, which are the same values of $N$ used in Fig. 4. Notice that $\zeta$ remains virtually stationary with $\phi$ from $\phi=0$ up to $45^{\circ}$ but descends steeply toward 0 as grazing incidence is approached. Equation (14) may be obtained, without performing any differentiation, by finding $\rho_{t}$ at the point of tangency $T$ in Fig. 3, solving Eq. (1) for the associated $X$,

$$
X=\left[\rho_{t}\left(1-r_{s}^{2}\right)-\left(1-r_{p}^{2}\right)\right] /\left[\rho_{t} r_{p}^{2}\left(1-r_{s}^{2}\right)-r_{s}^{2}\left(1-r_{p}^{2}\right)\right]
$$

and determining $\zeta$ by $\zeta=-\arg X / 2 \pi$.

Before concluding this section, we show a complementary family of constant thickness contours (CTCs) of the complex function $\rho_{t}$ of Eq. (1) for the same glass film in air $(N=1.5)$. Ten such curves appear in Fig. 7 


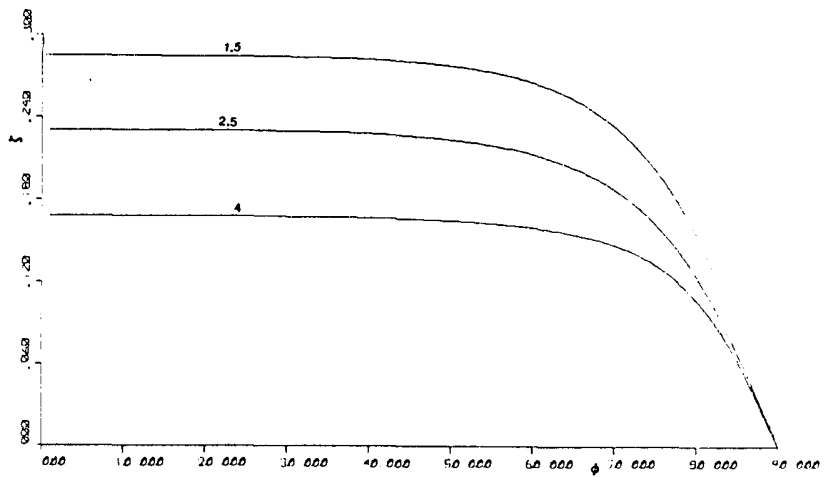

Fig. 6. Least normalized film thickness $\zeta$, which is required to produce the maximum phase retardance values indicated in Fig. 4, plotted vs the angle of incidence $\phi$.

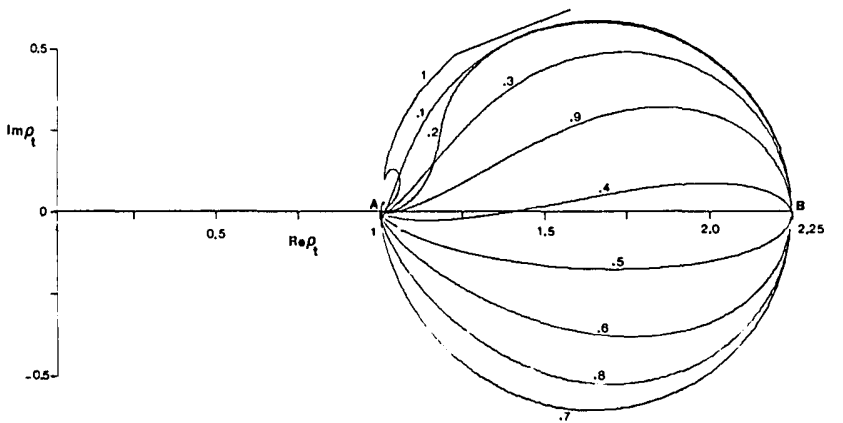

Fig. 7. Family of constant thickness contours (CTCs) of the transmission ellipsometric function $\rho_{t}$ in the complex plane for a glass film $(N=1.5)$ in air. The film thickness as a fraction of $\lambda / 2$ takes values from 0.1 to 1 in steps of 0.1 .

that correspond to setting $m=d /(\lambda / 2)=0.1$ to 1 in equal steps of 0.1 . Each curve is generated by increasing $\phi$ from 0 (point $A$ ) to $90^{\circ}$ (point $B$ ). The CTC for $m$ $=1$ shows a peculiar behavior and signals the onset of more varied CTCs at larger values of $m$. For certain values of $m$ in the chosen range (e.g., at 0.4 ) the CTC intersects the real axis and indicates the existence of an AOI at which linearly polarized light is transmitted linearly polarized through the tilted slab. For an optically thick film (e.g., $m>10$ ), several such intersections with the real axis occur between $A$ and $B$, indicating an oscillatory behavior of the phase retardance $\Delta_{t}$ with $\phi$. Such angles can be determined by a PSA (polarizer-sample-analyzer) null measurement and provide a unique characterization of the film refractive index and thickness. The procedure is similar to one described previously ${ }^{7}$ for determining $N$ and $d$ of a transparent film on a transparent substrate from the angles of incidence of zero reflection-induced ellipticity and is not repeated here. Finally, Fig. 7 also indicates that appreciable phase retardances $\Delta_{t}$ are possible only at certain film thicknesses (e.g., $m=0.1$ and $0.7)$ which is consistent with Fig. 6.

\section{Explicit Inversion for $\mathbf{N}$ and $\boldsymbol{d}$ in Transmission Ellipsometry} ting

A separate equation for $N$ alone is obtained by set-

$$
|X|=1 \text {, }
$$

where $X$ is given by the right-hand side of Eq. (15a). At a general AOI $\phi$, the resulting equation can only be solved numerically by iteration. The procedure is similar to that encountered in conventional ellipsometry of transparent thin films on transparent or absorbing substrates. . $^{2,8}$

Considerable simplification and an analytical solution are achieved at $45^{\circ} \mathrm{AOI}$. At this special angle the Fresnel interface reflection coefficients are interrelated by ${ }^{9} r_{p}=r_{s}^{2}$, and Eqs. (15a) and (15b) reduce to

$$
\left|\rho_{t}-\left(1+R_{s}\right)\right|=\left|R_{s}^{2} \rho_{t}-R_{s}\left(1+R_{s}\right)\right| \text {, }
$$

where

$$
R_{s}=r_{s}^{2} \text {, }
$$

which is the intensity reflectance of the ambient-film interface for the $s$ polarization at $45^{\circ} \mathrm{AOI}$. If we substitute

$$
\begin{aligned}
\rho_{t} & =a+j b, \\
& =\tan \psi_{t}\left(\cos \Delta_{t}+j \sin \Delta_{t}\right),
\end{aligned}
$$

where $\psi_{t}$ and $\Delta_{t}$ are the transmission ellipsometric parameters, into Eq. (16), the following simple quadratic equation is obtained:

$$
R_{s}^{2}-2 \mu R_{s}+1=0 \text {, }
$$

in which

$$
\mu=(a-1) /\left[(a-1)^{2}+b^{2}\right] .
$$

The solution of Eq. (20) is

$$
R_{s}=\mu \pm\left(\mu^{2}-1\right)^{1 / 2} \text {, }
$$

and only the root for which $0<R_{s}<1$ is accepted. Finally, $N$ is obtained from $R_{s}$ by ${ }^{1}$

$$
N=\left(1+R_{s}\right)^{1 / 2} /\left(1+R_{s}^{1 / 2}\right) \text {. }
$$

Once $N$ is determined, we can go back to Eq. (15a) and calculate complex $X$, and the film thickness $d$ :

$$
d=\{[-\arg X /(2 \pi)]+m\} D_{\phi},
$$

where $m$ is an integer. Note that $d$ can be determined from a single TELL measurement at a single AOI only up to an integral multiple $m$ of the film thickness period $D_{\phi}$. To fix $d$ unambiguously, measurement at a second AOI or wavelength is needed.

\section{Transmission Differential Phase Shift of an Ultrathin Film and the Experimental Verification of its Angular Dependence for a Soap Film}

The case of an ultrathin film with $\zeta$ or $(d / \lambda) \ll 1$ is of particular interest. For such film, $X=1-j 2 \pi \zeta$, to first order in $\zeta$. Substitution of this linearized expression for $X$ into Eq. (1) gives $\rho_{t}=1+j \Delta_{t}$, where

$$
\left.\Delta_{t}=2 \pi \zeta\left[r_{s}^{2} /\left(1-r_{s}^{2}\right)\right]-\left[r_{p}^{2} /\left(1-r_{p}^{2}\right)\right]\right\} \text {, }
$$

and the associated $\psi_{t}=\pi / 4$, all to first order in $\zeta$. Replacing $r_{p}$ and $r_{s}$ by their standard expressions ${ }^{2}$ and introducing $d / \lambda$ instead of $\zeta$ [Eqs. (3), (6), and (7)], transform Eq. (25) into the more explicit form

$$
\Delta_{l} /(2 \pi)=\left[\left(N_{1}^{2}-N_{0}^{2}\right)^{2} / 2 N_{0} N_{1}^{2}\right](\sin \phi \tan \phi)(d / \lambda) .
$$




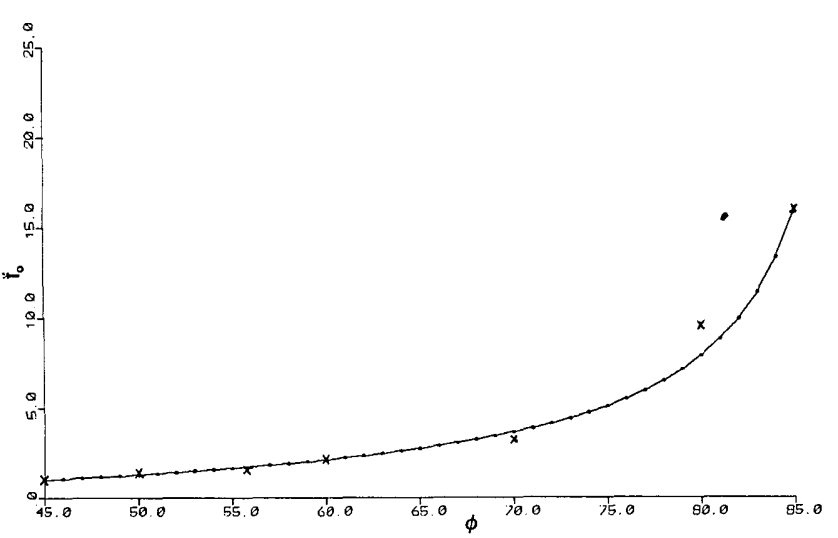

Fig. 8. Obliquity factor $f_{o}(\phi)$ as a function of the angle of incidence $\phi$. The continuous line is calculated from Eq. (28), whereas the points marked by $X$ are obtained from ellipsometric measurements of the transmission phase retardance of a stable planar soap film in air at $\lambda=633 \mathrm{~nm}$.

The proportionality of $\Delta_{t}$ with $d / \lambda$ is anticipated.

To emphasize the angular dependence of $\Delta_{t}$, we introduce an obliquity factor defined by

$$
\begin{aligned}
f_{o}(\phi) & =\Delta_{t}(\phi) / \Delta_{t}\left(45^{\circ}\right), \\
& =2^{1 / 2} \sin \phi \tan \phi .
\end{aligned}
$$

We tested the validity of this theoretical expression, Eq. (28), by performing TELL on a stable planar soap film ${ }^{10}$ in air. The film is supported by a small-diam $(\sim 1 \mathrm{~cm})$ copper $O$ ring and null ellipsometry is carried out at multiple angles of incidence, $\phi=45,50,60,70$, 80 , and $85^{\circ}$, using a $633-\mathrm{nm} \mathrm{He}-\mathrm{Ne}$ laser as a source and a Gaertner ellipsometer arranged in the straight through configuration. The results appear in Fig. 8 in which the continuous line represents Eq. (28) and the crosses $(X)$ are the experimental points. The measured absolute values of $\Delta_{t}$ at 45 and $85^{\circ}$ are 0.35 and $5.59^{\circ}$, and the theoretical and experimental values of the obliquity factor $f_{o}\left(85^{\circ}\right)$ are 16.10 and 15.97 , respectively. The agreement of theory and experiment is satisfactory.

A peculiarity of Eq. (28) is that it predicts a singularity at grazing incidence. Such a singularity does not exist; it merely indicates the failure of the linear approximation in the immediate neighborhood of grazing incidence. In fact, $\Delta_{t}$ reaches a maximum near grazing incidence before it drops to 0 at exactly $\phi=90^{\circ}$. The true behavior of $\Delta_{t}$ with $\phi$ at large angles $\left(80 \leq \phi \leq 90^{\circ}\right)$ is illustrated in Fig. 9 for a thin film with $N=1.5$ and $d /$ $\lambda=0.01$ and is calculated based on the exact Eq. (1). Maximum $\Delta_{t}$ occurs at $\sim 1^{\circ}$ away from grazing incidence and has a value of $\sim 22.5^{\circ}$ or the equivalent of 1 / 16 th wave.

The dependence of $\Delta_{t}$ on the refractive index of the film $N$ (assuming that light is incident from air, $N_{0}=1$ ) is given by the factor

$$
\begin{aligned}
f_{n}(N) & =\left[\Delta_{t}\left(45^{\circ}\right) / 2 \pi\right] /(d / \lambda), \\
& =\left(1 / 2^{1.5}\right)[N-(1 / N)]^{2},
\end{aligned}
$$

and is plotted as a function of $N$ in Fig. 10. According

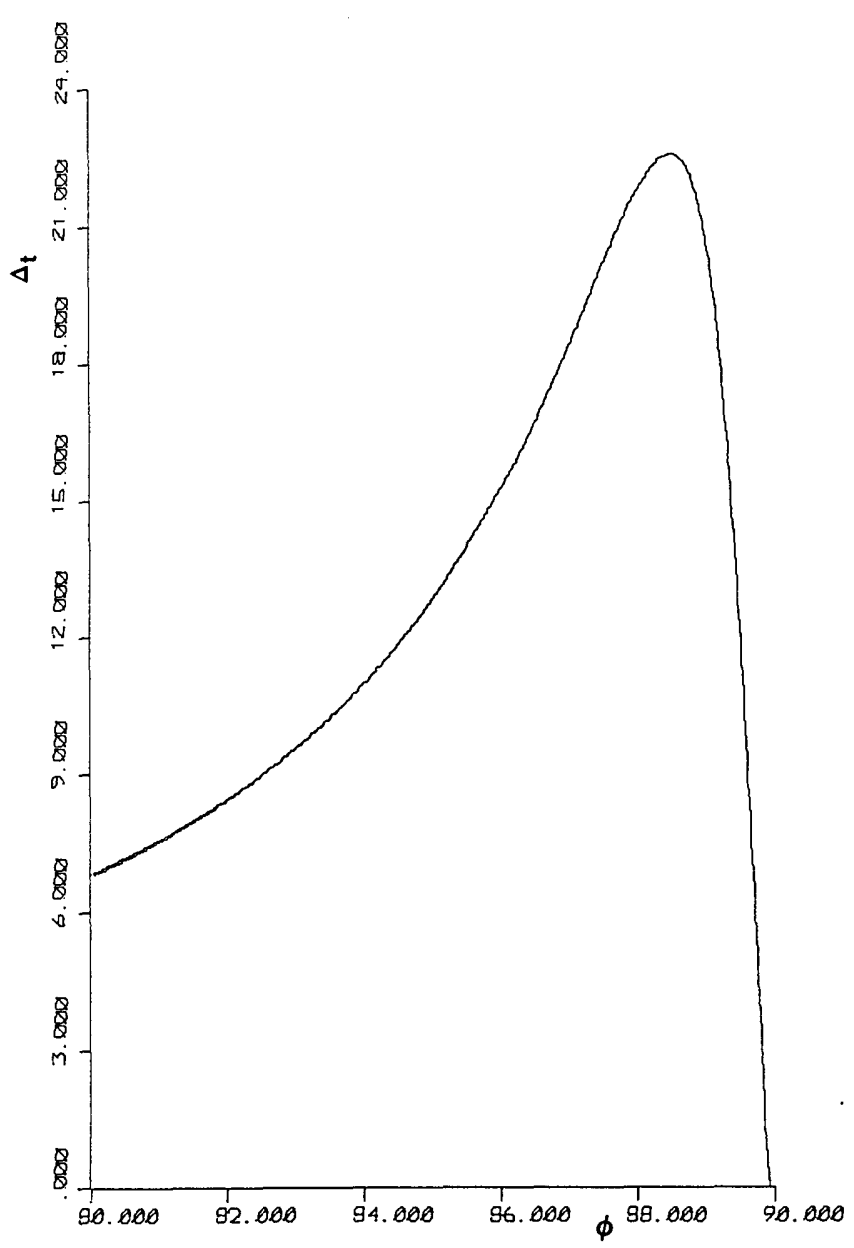

Fig. 9. Transmission phase retardance, $\Delta_{t}$, at large angles of incidence, $80 \leq \phi \leq 90^{\circ}$, for a glass film $(N=1.5)$ with $d / \lambda=0.01$ in air. Instead of the singularity predicted by the obliquity factor, Eq. (28), $\Delta_{t}$ reaches a maximum at an angle $\sim 1^{\circ}$ away from grazing incidence and drops to 0 at exactly $\phi=90^{\circ}$.

to this index factor, a Ge thin film $(N=4)$ produces an $\sim 20$ times larger differential transmission phase shift than a glass film $(N=1.5)$ for the same $d / \lambda$ (which is $\ll$ 1) at $45^{\circ}$ and over most of the range of $\phi\left(0<\phi<75^{\circ}\right)$. Values of $N<1$ are allowed in Fig. 10 to represent the situation of partial internal reflection.

For an ultrathin film, such as the molecular bilayer soap film, variable angle TELL does not permit the separation of $N$ and $d / \lambda$, as is evident from Eq. (26). In essence, measurement at one angle foretells the results of measurements at other angles, as the obliquity factor dictates. One way to determine $N$ separately is to measure the Brewster angle $\phi_{B}$ of minimum $p$ reflectance. For the soap film in air this gives $\phi_{B}=55.78^{\circ}$ and $N=1.47$. (TELL at $\phi_{B}$ provides an extra point at that angle that appears in Fig. 8.) From this value of $N$ and the measured $\Delta_{t}$ (e.g., $0.35^{\circ}$ at $\left.45^{\circ} \mathrm{AOI}\right), d / \lambda$ is estimated using Eq. (26) to be $4.41 \times 10^{-3}$; hence $d=$ $2.8 \mathrm{~nm}$ and the molecular dimension $=1.4 \mathrm{~nm}$.

In the foregoing analysis, the uniaxial optical anisotropy or birefringence of the soap film, caused by the molecular orientation in the direction normal to the film boundaries, is neglected. 


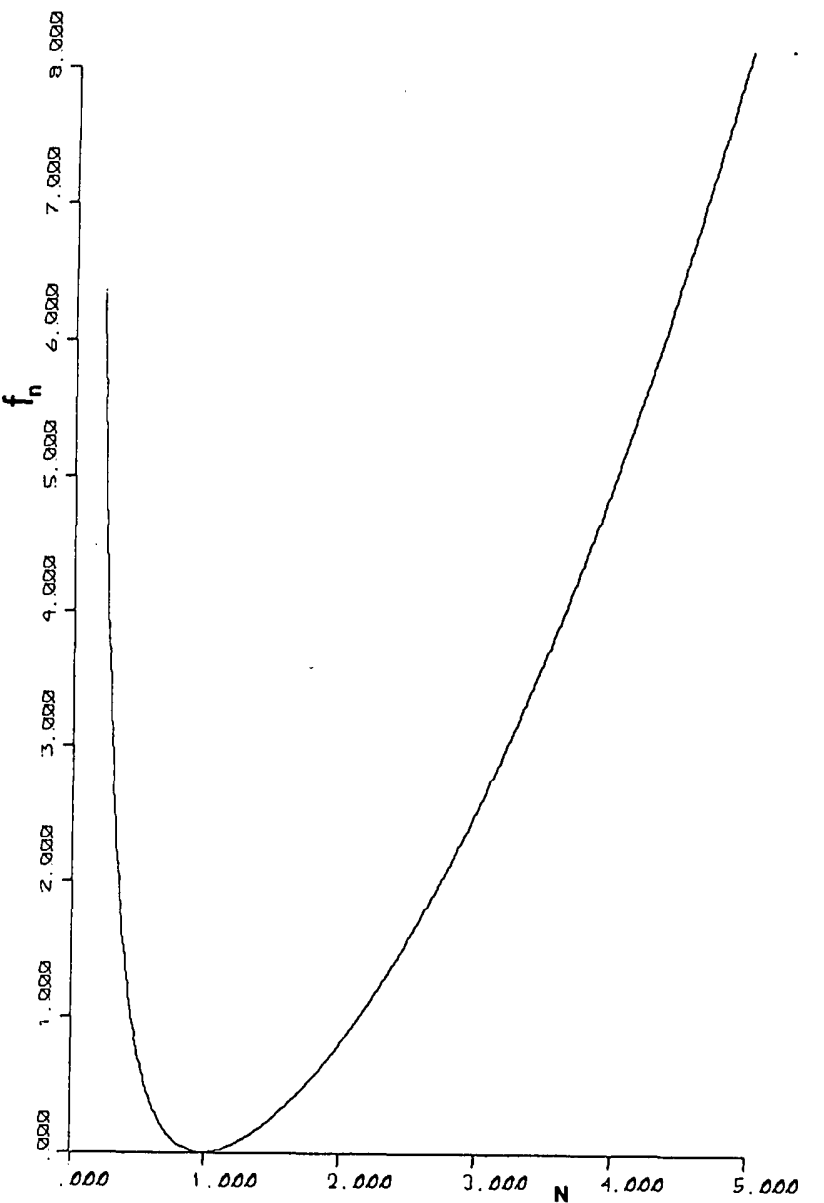

Fig. 10. Index factor, Eq. (30).

\section{Summary}

A transparent unbacked (pellicle) or embedded thin film provides an interesting object for transmission ellipsometry (TELL). TELL alone (with no measurements in reflection) is sufficient to determine the refractive index and thickness $(N, d)$ of the film. An explicit analytical method for determining $N$ and $d$ from TELL at $45^{\circ}$ incidence is given. An expression for the transmission phase retardance $\Delta_{t}$ in light passing through an ultrathin film $(d / \lambda \ll 1)$ is derived in product form that clearly separates the dependence of $\Delta_{t}$ on incidence angle $\phi$ (an obliquity factor that varies as $\sin \phi \tan \phi)$ and film index $N$. TELL measurements on a stable soap film in air have confirmed the validity of the theoretical obliquity factor. Variable angle TELL on an ultrathin film does not permit the separation of $N$ and $d / \lambda$. However, this ambiguity is easily resolved by a Brewster angle measurement of $N$. (Alternatively, combined reflection and TELL can be used as described in Ref. 1.) Finally, the relations between the ellipsometric parameters of reflection and transmission by the same film at the same angle are given in the following appendix.

\section{Appendix}

The ratio of complex reflection coefficients for the $p$ and $s$ polarizations $\rho_{r}$ is related to $\rho_{t}$ by ${ }^{1}$

$$
\rho_{r}=G(N, \phi) \rho_{t},
$$

where

$$
G(N, \phi)=\left(r_{p} / r_{s}\right)\left[\left(1-r_{s}^{2}\right) /\left(1-r_{p}^{2}\right)\right]
$$

is real because we have excluded total internal reflection. In the Nebraska (Muller) conventions, ${ }^{11}$ which we follow, $G<0$ for $\phi<\phi_{B}$ and $G>0$ for $\phi>\phi_{B}$, where $\phi_{B}$ is the Brewster angle. Consequently, from Eq. (A1), the reflection phase retardance $\Delta_{r}$ is simply related to the transmission phase retardance $\Delta_{t}$ by

$$
\begin{aligned}
& \Delta_{r}=\Delta_{t} \pm \pi, \quad \phi<\phi_{B}, \\
& \Delta_{r}=\Delta_{t}, \quad \phi>\phi_{B} .
\end{aligned}
$$

Thus, for an unbacked or embedded transparent thin film, $\Delta_{r}$ and $\Delta_{t}$ are not independent and it suffices to measure only one of them. ${ }^{12}$ The retardance $\Delta_{t}$ is easier to measure because the transmitted beam is more intense and passes through the film with no angular deviation. The diattenuations in reflection and transmission are related by

$$
\tan \psi_{r}=|G| \tan \psi_{t},
$$

where $\psi_{i}=\arctan \left|\rho_{i}\right|(i=r$ or $t)$ is the second ellipsometric parameter, as usual.

\section{References}

1. R. M. A. Azzam, "Simple and Direct Determination of Complex Refractive Index and Thickness of Unsupported or Embedded Thin Films by Combined Reflection and Transmission Ellipsometry at $45^{\circ}$ Angle of Incidence," J. Opt. Soc. Am 73, 1080-1082 (1983); "Ellipsometry of Unsupported or Embedded Thin Films," J. Phys. (Paris) Colloq. 44, C10-67-C10-70 (1983).

2. R. M. A. Azzam and N. M. Bashara, Ellipsometry and Polarized Light (North-Holland, Amsterdam, 1987).

3. See, for example, W. R. LePage, Complex Variables and the Laplace Transform for Engineers (Dover, New York, 1961), Sec. 3.10.

4. R. M. A. Azzam, "Polar Curves for Transmission Ellipsometry," Opt. Commun. 14, 145-147 (1975).

5. See, for example, A. E. Taylor, Calculus with Analytic Geometry (Prentice Hall, Englewood Cliffs, NJ, 1959), Ch. 7.

6. D. A. Holmes, "Wave Optics Theory of Rotary Compensators," J. Opt. Soc. Am. 54, 1340-1347 (1964).

7. R. M. A. Azzam and A.-R. M. Zaghloul, "Determination of the Refractive Index and Thickness of a Transparent Film on a Transparent Substrate from the Angles of Incidence of Zero Reflection-Induced Ellipticity," Opt. Commun. 24, 351-354 (1978).

8. A. R. Reinberg, "Ellipsometer Data Analysis with a Small Programmable Desk Calculator," Appl. Opt. 11, 1273-1274 (1972).

9. F. Abelès, "Un Théoreme relatif à la réflexion metallique," C. R. Acad. Sci. 220, 1942-1943 (1950).

10. Wonder Bubbles, Chemtoy Corp., Chicago, IL 60624. At normal room temperature and pressure, the stable film can stay intact, without rupture, for $15 \mathrm{~min}$ or longer.

11. R. H. Muller, "Definitions and Conventions in Ellipsometry," Surf. Sci. 16, 14-33 (1969).

12. This is not the case for a partially transmitting thin film with $k$ $\neq 0$. Even with a numerically small $k$ value (e.g., 0.01 ), the difference $\Delta_{r}-\Delta_{t}$ assumes anomalously large values in the immediate neighborhood of the pseudo-Brewster angle. 